\title{
THE COHOMOLOGY OF GROUP EXTENSIONS
}

\author{
BY \\ L. S. CHARLAP(1) AND A. T. VASQUEZ(2)
}

0 . Introduction. If $G$ is a group with a finitely generated normal abelian subgroup $K$, then it is well known that the isomorphism class of $G$ is determined by (A) the groups $K$ and $G / K$, (B) the structure of $K$ as a module over $G / K$, and (C) a cohomology class $\alpha \in H^{2}(G / K ; K)$. In principle then, it should be possible to compute $H^{*}(G)$, the cohomology ring of $G$, from the above information. Practically, however, this seems to be impossible in general, even if we assume that the cohomology of $K$ and $G / K$ is known.

The Hochschild-Serre spectral sequence [7] provides us with a sequence of differential rings $\left(E_{r}, d_{r}\right)(r=1,2, \cdots)$ which approximate the ring $H^{*}(G)$ and such that $E_{r+1}=H\left(E_{r}, d_{r}\right)$. Hochschild and Serre computed $E_{2}$ and found that $E_{2}^{p, q} \approx H^{p}\left(G / K ; H^{q}(K)\right)$. So $E_{2}$ depends only on (A) and (B) and is therefore a rather crude approximation to $H^{*}(G)$. We determine $d_{2}$ (and hence $E_{3}$ ) and show that it depends not only on (A) and (B), but also on (C).

If $A$ is a $G / K$-module, it can be considered a $G$-module, and we let $E_{r}(A)$ denote the spectral sequence associated with $H^{*}(G ; A)$. We assume $\boldsymbol{F}$ is a ring with some "nice" properties (a field will suffice). Then $H_{i}(K ; F)$ and $H^{i}(K ; F)$ are $G / K$-modules. We fix an integer $N$ and write $\hat{E}_{r}=E_{r}\left(H_{N}(K ; \boldsymbol{F})\right) ; \bar{E}_{r}=E_{r}\left(H^{N}(K ; \boldsymbol{F})\right)$, and $E_{r}=E_{r}(F)$. The hypothesis on $F$ implies $H^{N}(K ; F) \approx \operatorname{Hom}_{F}\left(H_{N}(K ; F), F\right)$ and so we have a pairing $\bar{E}_{r}^{p, q} \otimes \hat{E}_{r}^{p^{\prime}, q^{\prime}} \rightarrow E_{r}^{p+p^{\prime}, q+q^{\prime}}$. There is also a canonical isomorphism $\theta: E_{2}^{p, N} \rightarrow \bar{E}_{2}^{p, 0}$ since

$$
H^{p}\left(G / K ; H^{N}(K ; \boldsymbol{F})\right) \approx H^{p}\left(G / K ; H^{0}\left(K ; H^{N}(K ; \boldsymbol{F})\right)\right) .
$$

Our main theorem says that to find $d_{2}$ of an element $u \in E_{2}^{p, N}$, one takes the product of $\theta(u) \in E_{2}^{p, 0}$ with an element $x^{N} \in E_{2}^{2, N-1} \approx H^{2}\left(G / K ; H^{N-1}\left(K ; H_{N}(K ; F)\right)\right)$. We now proceed to describe this element $x^{N}$.

$x^{N}$ can be written as $\hat{d}_{2}$ of an element $f^{N} \in \hat{E}_{2}^{0, N} \approx H^{0}\left(G / K ; H^{N}\left(K ; H_{N}(K ; F)\right)\right)$. $f^{N}$ is that $G / K$-invariant element of $H^{N}\left(K ; H_{N}(K, F)\right)$ which corresponds to the identity map under the isomorphism $H^{N}\left(K ; H_{N}(K ; F)\right) \approx \operatorname{Hom}_{F}\left(H_{N}(K ; F)\right.$,

Received by the editors May 22, 1964.

(1) Supported by Air Force (AF-49(638)-253) and National Science Foundation (GP780).

(2) Supported by National Science Foundation postdoctoral fellowship and NSF grants (GP779) and (GP780). 
$H_{N}(K ; F)$ ). Now to indicate the dependence of $\hat{d}_{2}$ on $\alpha$, we put $D_{\alpha}=\hat{d}_{2}$ (recall $\alpha \in H^{2}(G / K ; K)$ classifies the extension $\left.G\right)$. So we can think of $D_{\alpha}$ as

$$
D_{\alpha}: H^{p}\left(G / K ; H^{q}\left(K ; H_{N}(K ; F)\right)\right) \rightarrow H^{p+2}\left(G / K ; H^{q-1}\left(K ; H_{N}(K ; F)\right)\right) .
$$

Note that if we consider the spectral sequence for the split extension (i.e. the case $\alpha=0$ ), we get a map $D_{0}$ with the same range and domain as $D_{\alpha}$ (since $\hat{E}_{2}$ is independent of $\alpha$ ). We then show that

$$
D_{\alpha}\left(f^{N}\right)=D_{0}\left(f^{N}\right)-\boldsymbol{P}_{*}(\alpha)
$$

where $D_{0}\left(f^{N}\right)$ is, of course, independent of $\alpha$ and $\boldsymbol{P}_{*}$ can be described as follows: Since $K$ is abelian, Pontryagin multiplication in $H_{*}(K, \boldsymbol{F})$ yields a map $\boldsymbol{P}$ which fits in the following composition

$$
\begin{aligned}
K & \left.\approx H_{1}(K ; Z) \stackrel{\chi_{*}}{\rightarrow} H_{1}(K, \boldsymbol{F}) \stackrel{\boldsymbol{P}}{\rightarrow} \operatorname{Hom}_{F}\left(H_{N-1}(K ; \boldsymbol{F}), H_{N}(K ; \boldsymbol{F})\right)\right) \\
& \approx H^{N-1}\left(K ; H_{N}(K ; \boldsymbol{F})\right)
\end{aligned}
$$

where $\chi$ is the map that sends 1 in $Z$ to 1 in $\boldsymbol{F}$. Then $\boldsymbol{P}_{*}$ is the induced map

$$
\boldsymbol{P}_{*}: H^{i}(G / K ; K) \rightarrow H^{i}\left(G / K ; H^{N-1}\left(K ; H_{N}(K ; \boldsymbol{F})\right)\right) \text {. }
$$

Recapitulating, we state

MAIN THEOREM. Let $u \in E_{2}^{p, N}$. Then

$$
d_{2}(u)=(-1)^{p} \theta(x) \cdot\left[D_{0}\left(f^{N}\right)-\boldsymbol{P}_{*}(\alpha)\right] .
$$

The proof of this theorem occupies the first five sections.

$\S 7$ investigates the classes $v^{N}=D_{0}\left(f^{N}\right)$, which we call the characteristic classes of the $G / K$ module $K$. We reprove in passing a fact noted by Hochschild and Serre; in our notation it asserts that $v^{1}=0$. A description of $v^{N}$ is given which owes nothing to the notion of a spectral sequence. Examples of the theory are discussed in $\S 8$. $\$ 8$ contains no proofs; the proofs will appear in [9]. In $\$ 9$ the Main Theorem is applied to the study of an interesting class of groups arising from the study of Riemannian geometry. These groups are characterized by the fact that $K$ is free abelian and maximal abelian in $G, G / K$ is cyclic of prime order, and $G$ is torsion free.

We wish to thank Armand Borel for several stimulating conversations, and the referee for many helpful comments.

1. Preliminaries. Let $G$ be a group with a finitely generated normal abelian subgroup $K$, and let $\Phi$ equal $G / K$. This can be summarized by the exact sequence

$$
0 \rightarrow K \stackrel{j}{\rightarrow} G \stackrel{\rho}{\rightarrow} \Phi \rightarrow 1 .
$$

We have written 0 and the left and 1 on the right to denote the trivial group; 
the notation is so chosen because we will use additive notation for $K$ and multiplicative notation for $\Phi$.

It is well known that in this situation $K$ is a left $\Phi$-module and the extension $\left(^{*}\right)$ is classified by an element $\alpha \in H^{2}(\Phi ; K)$ (cf. [3, p. 302]). In fact, $G$ may be represented as pairs $(k, \sigma)$ with $k \in K$ and $\sigma \in \Phi$. The multiplication in $G$ is then given by

$$
\left(k_{1}, \sigma_{1}\right) \cdot\left(k_{2}, \sigma_{2}\right)=\left(k_{1}+\sigma_{1} \cdot k_{2}+a\left(\sigma_{1}, \sigma_{2}\right), \sigma_{1} \sigma_{2}\right)
$$

where $a$ is a nonhomogeneous normalized 2-cycle representing $\alpha$. The homomorphisms $j$ and $\rho$ are then described by

$$
j(k)=(k, 1) \text { for } k \in K,
$$

and

$$
\rho(k, \sigma)=\sigma \text { for } \sigma \in \Phi .
$$

We recall here the construction of the double complex which leads to the Hochschild-Serre spectral sequence (see Chapter I in [8] for more details). Let $A$ be a left $G$-module and $C_{*}(G)$ the standard homogeneous complex for $G$. Let $U^{*}$ be the cochain complex with $U^{j}=\operatorname{Hom}_{K}\left(C_{j}(G), A\right)$ and with the obvious coboundary operator. Then $U^{j}$ is a left $\Phi$-module according to the rule

$$
(\sigma \cdot f)(c)=g \cdot\left[f\left(g^{-1} \cdot c\right)\right] \text { for } f \in U^{j}, c \in C_{j}(G) \text { and } \sigma \in \Phi \text { and } g \in \rho^{-1}(\sigma) \subset G \text {. }
$$

Define $L^{p, q}=C^{p}\left(\Phi, U^{q}\right)$, the group of nonhomogeneous cochains of $\Phi$ with values in $U^{q}$, and let $L=\bigoplus_{p, q} L^{p, q}$. $L$ has two coboundary operators

and

$$
d_{\Phi}: L^{p, q} \rightarrow L^{p+1, q}
$$

$$
d_{u}: L^{p, q} \rightarrow L^{p, q+1}
$$

which are defined in the obvious fashion. As usual, we set

$$
d=d_{\Phi}+(-1)^{p} d_{u}: L^{p, q} \rightarrow L^{p+1, q} \oplus L^{p, q+1},
$$

which makes $L$ a cochain complex. The spectral sequence in question arises from the filtration of $L$ by the groups

$$
L_{i}=\bigoplus_{q=0}^{\infty} L_{i}^{q} \quad \text { where } L_{i}^{q}=\bigoplus_{p \geqq i} L^{p, q} .
$$

Now consider $E_{2}^{p, q}(A)$. According to $\left[7\right.$, p. 87], an element $x \in E_{2}^{p, q}(A)$ can be represented as

such that

$$
x^{p, q}+x^{p+1, q-1} \in L^{p, q} \oplus L^{p+1, q-1}
$$

$$
\begin{aligned}
& d_{u} x^{p, q}=0 \\
& d_{\Phi} x^{p, q}=(-1)^{p} d_{u} x^{p+1, q-1} .
\end{aligned}
$$


Furthermore $x$ is 0 in $E_{2}^{p, q}(A)$ iff there is $y^{p, q-1}+y^{p-1, q} \in L^{p, q-1} \otimes L^{p-1, q}$ such that

$$
\begin{aligned}
d_{\Phi} y^{p-1, q}+(-1)^{p} d_{u} y^{p, q-1} & =x^{p, q} \\
d_{u} y^{p-1, q} & =0 .
\end{aligned}
$$

We need to isolate the following fact:

REMARK. Let $x \in E_{2}^{p, q}(A)$ be represented by $x^{p, q}+x^{p+1, q-1}$ as above. Then $d_{2}(x) \in E^{p+2, q-1}(A)$ can be represented by

$$
d_{\Phi} x^{p+1, q-1}+0 \in L^{p+2, q-1} \oplus L^{p+3, q-2} .
$$

This is clear from the discussion in [7].

We will also have need of the isomorphism

$$
\phi: E_{2}^{p, q}(A) \approx H^{p}\left(\Phi ; H^{q}(K ; A)\right),
$$

and the two following descriptions of $H^{q}(K ; A)$ : Let $V^{j}=\operatorname{Hom}_{K}\left(C_{j}(K), A\right)$ and $V^{*}=\bigoplus_{j} V^{j}$. Then $H^{q}(K ; A) \approx H^{q}\left(V^{*}\right)$ and this is the usual homogeneous cochain representation. However, if $U^{*}$ is as defined above, we also have $H^{q}(K ; A) \approx H^{q}\left(U^{*}\right)$, and since the crux of the main argument is a comparison of these two descriptions of $H(K ; A)$, we will go into it in some detail.

Let $\varepsilon: C_{0}(K) \rightarrow Z$ and $\eta: C_{0}(G) \rightarrow Z$ be the usual augmentations. With these augmentations both $C_{*}(K)$ and $C_{*}(G)$ are $Z[K]$-free acyclic resolutions of $Z$; hence by general theory, they are homotopically equivalent. Using our description of $G$ as pairs $(k, \sigma)$, we pick a specific equivalence as follows: The homomorphism $j: K \rightarrow G: k \rightarrow(k, 1)$ yields a chain map $j_{*}: C_{*}(K) \rightarrow C_{*}(G)$. Define $\psi_{*}: C_{*}(G) \rightarrow C_{*}(K)$ by

$$
\psi_{n}\left(\left(k_{0}, \sigma_{0}\right), \cdots,\left(k_{n}, \sigma_{n}\right)\right)=\left(k_{0}, \cdots, k_{n}\right) .
$$

Then $\psi_{n} j_{n}$ is the identity, and it follows that $j_{n} \psi_{n}$ is homotopic to the identity. However a trivial modification of the usual proof gives us the following more convenient proposition.

Proposition 1.1. There is a chain homotopy $s_{n}: C_{n}(G) \rightarrow C_{n+1}(G)$ between $j_{n} \psi_{n}$ and the identity which satisfies

$$
s_{n} j_{n}=0 .
$$

As we remarked above, $U^{*}$ is a left $\Phi$-module, and this makes $H^{*}(K ; A)\left(\approx H^{*}\left(U^{*}\right)\right)$ also a left $\Phi$-module. It is shown in [8] (using slightly different terminology) that if we think of $H^{*}(K ; A)$ as $H^{*}\left(V^{*}\right)$, this $\Phi$-action can be described as follows: Let $u \in H^{j}(K ; A)$ be represented by $f \in V^{j}$, and let $g \in G$ and $\sigma=\rho(g) \in \Phi$. Then $\sigma \cdot u$ is represented by $f^{\prime} \in V^{j}$ where

$$
f^{\prime}\left(k_{0}, \cdots, k_{j}\right)=g \cdot\left[f\left(\sigma^{-1} \cdot k_{0}, \cdots, \sigma^{-1} \cdot k_{j}\right)\right] \in A .
$$


Now if the action of $G$ on $A$ is such that $K$ acts trivially on $A$, then we can write $\sigma \cdot f$ for $f^{\prime}$, and (3) makes $V^{*}$ into a left $\Phi$-module. From now on we assume that $K$ acts trivially on $A$.

One must be careful in comparing the left $\Phi$-modules $V^{*}$ and $U^{*}$ by means of the maps $j^{*}$ and $\psi^{*}$.

Proposition 1.2. Let $f \in V^{n}$. Then $\sigma \cdot\left(\psi_{n}^{*} f\right) \in U^{n}$ and

$$
j_{n}^{*}\left[\sigma \cdot\left(\psi_{n}^{*} f\right)\right]=\sigma \cdot f \in V^{n} .
$$

The proof consists of checking the obvious statements and will be omitted. However, it is important to note that the analogous statement for $F \in U^{n}$ is not true.

2. A reduction of the problem. Let $\mathbf{F}$ be a principal ideal domain considered as a trivial $G$-module. In the applications $\mathbf{F}$ will be a field or the integers. We want to compute

$$
d_{2}: E_{2}^{p, q}(F) \rightarrow E_{2}^{p+2, q-1}(F) .
$$

By a technical device, we can reduce this to a special case.

We first fix an integer $N \geqq 0$ and then introduce two new spectral sequences which correspond to two choices for coefficients. We recall that $H^{N}(K ; F)$ is a left $\Phi$-module; in an obvious fashion so is $H_{N}(K ; F)$, and both can be considered as left $G$-modules on which $K$ acts trivially. We now consider the spectral sequences associated with the cohomology of $G$ with these coefficients. We put

$$
\begin{aligned}
& \bar{E}_{r}^{p, q}=E_{r}^{p, q}\left(H^{N}(K ; F)\right), \\
& \hat{E}_{r}^{p, q}=E_{r}^{p, q}\left(H_{N}(K ; F)\right), \\
& E_{r}^{p, q}=E_{r}^{p, q}(F) \quad \text { all for } p, q \geqq 0 \text { and } r \geqq 2 .
\end{aligned}
$$

Note that the above simplified notation ignores the choice of $N$. We hope this will cause no serious confusion.

The universal coefficient theorem yields the following split exact sequence:

$$
0 \rightarrow \operatorname{Ext}_{F}\left(H_{N-1}(K ; F), F\right) \rightarrow H^{N}(K ; F) \rightarrow \operatorname{Hom}_{F}\left(H_{N}(K ; F), F\right) \rightarrow 0 .
$$

This gives us a pairing $H^{N}(K ; F) \otimes H_{N}(K ; F) \rightarrow F$, and we denote the image of $u \otimes a$ by $\langle u, a\rangle \in \boldsymbol{F}$ for $u \in H^{N}(K ; F)$ and $a \in H_{N}(K ; \boldsymbol{F})$. If we let $\Phi$ act diagonally on the tensor product, the pairing is a left $\Phi$-module homomorphism, i.e.

$$
\langle\sigma \cdot u, \sigma \cdot a\rangle=\langle u, a\rangle \text { for } \sigma \in \Phi .
$$

It is standard that this pairing gives a pairing of spectral sequences

$$
\bar{E}_{r}^{p, q} \otimes \hat{E}_{r}^{p^{\prime}, q^{\prime}} \rightarrow E_{r}^{p+p^{\prime}, q+q^{\prime}}
$$

and we have the usual multiplicative formula 


$$
d_{r}(x \cdot y)=d_{r}(x) \cdot y+(-1)^{p+q} x \cdot \hat{d}_{r}(y)
$$

for $x \in \bar{E}_{r}^{p, q}$ and $y \in \hat{E}_{r}^{p^{\prime}, q^{\prime}}$. Note, however, that $\phi(x \cdot y)=(-1)^{q, p^{\prime}} \phi(x) \cdot \phi(y)$.

We define an isomorphism

$$
\theta: E_{2}^{p, N} \rightarrow \bar{E}_{2}^{p, 0}
$$

as follows: $H^{N}(K ; \boldsymbol{F})$ is a trivial $K$-module so $H^{0}\left(K ; H^{N}(K ; \boldsymbol{F})\right) \approx H^{N}(K ; \boldsymbol{F})$. Since

and

$$
E_{2}^{p, N} \approx H^{p}\left(\Phi ; H^{N}(K ; \boldsymbol{F})\right)
$$

$$
\hat{E}_{2}^{p, 0} \approx H^{p}\left(\Phi ; H^{0}\left(K ; H^{N}(K ; F)\right)\right.
$$

we can let $\theta$ be induced by this isomorphism.

Note that $H_{i}(K ; F)$ is an $F$-module. We now make the following definite restriction on $K$ and $\boldsymbol{F}$.

We assume that $H_{i}(K ; F)$ is $F$-free for all $i$.

This will be the case, for example, if $\boldsymbol{F}$ is a field, or if $K$ is free and $\boldsymbol{F}=Z$. Now

$$
H^{N}\left(K ; H_{N}(K ; \boldsymbol{F})\right) \approx \operatorname{Hom}_{\mathbf{F}}\left(H_{N}(K ; \boldsymbol{F}), H_{N}(K ; \boldsymbol{F})\right) .
$$

We let $g^{N} \in H^{N}\left(K ; H_{N}(K ; F)\right)$ correspond to the identity map: $H_{N}(K ; F) \rightarrow H_{N}(K ; F)$. It is easy to see that $g^{N}$ is left fixed under the action of $\Phi$ on $H^{N}\left(K ; H_{N}(K ; F)\right)$, and hence defines an element

$$
f^{N} \in E_{2}^{0, N} \approx H^{0}\left(\Phi ; H^{N}\left(K ; H_{N}(K ; F)\right)\right) .
$$

Proposition 2.1. Let $x \in E_{2}^{p, N}$. Then

$$
x=\theta(x) \cdot f^{N}
$$

Proof. We will use the pairing

$$
H^{p}\left(\Phi ; H^{0}\left(K ; H^{N}(K ; F)\right)\right) \otimes H^{0}\left(\Phi ; H^{N}\left(K ; H_{N}(K ; F)\right)\right) \rightarrow H^{p}\left(\Phi ; H^{N}(K ; F)\right) .
$$

There is no sign change to worry about since in comparing this pairing to $E_{2}^{p, 0} \otimes E_{2}^{0, N} \rightarrow E_{2}^{p, N}$, the sign change is $(-1)^{0+0}=+1$. The problem is now one of computing cup products in $H^{*}(\Phi ;)$, and the result follows from the fact that if $\tilde{u} \in H^{0}\left(K ; H^{N}(K, F)\right)$ corresponds to $u \in H^{N}(K ; F)$ and $\gamma \in H_{N}(K ; F)$, then

$$
\left\langle\tilde{u} \cdot g^{N}, \gamma\right\rangle=\langle u, \gamma\rangle
$$

and hence $\tilde{u} \cdot g^{N}=u$.

Proposition 2.2. $d_{2}(x)=(-1)^{p} \theta(x) \cdot d_{2}\left(f^{N}\right)$.

Proof. $d_{2}(x)=d_{2}\left(\theta(x) \cdot f^{N}\right)=d_{2}(\theta(x)) \cdot f^{N}+(-1)^{p+0} \theta(x) \cdot d_{2}\left(f^{N}\right)=0+(-1)^{p} \theta(x) \cdot d_{2}\left(f^{N}\right)$.

Now under the isomorphisms $E_{2}^{0, N} \approx H^{0}\left(\Phi ; H^{N} K ; H_{N}(K ; F)\right)$ and 


$$
\hat{E}_{2}^{2, N-1} \approx H^{2}\left(\Phi ; H_{N-1}\left(K ; H_{N-1}(K ; \boldsymbol{F})\right)\right),
$$

$d_{2}$ induces a homomorphism

$$
D_{\alpha}: H^{0}\left(\Phi ; H^{N}\left(K ; H_{N}(K ; \boldsymbol{F})\right)\right) \rightarrow H^{2}\left(\Phi ; H_{N-1}\left(K ; H_{N}(K ; \boldsymbol{F})\right)\right) .
$$

We emphasize the fact that the domain and range of $D_{\alpha}$ depend only on $K, \Phi$ and the action of $\Phi$ on $K$ while the homomorphism itself depends on the extension $G$ which is classified by $\alpha \in H^{2}(\Phi ; K)$. The next sections will be devoted to computing $D_{a}\left(f^{N}\right)$.

3. A computation. In this computation of $D_{\alpha}\left(f^{N}\right)$, no explicit use will be made of the special properties of $f^{N}$ other than the fact that it lies in $E_{2}^{0, N}(A)$ for some left $\Phi$-module $A$; so we will work in this generality. The basic difficulty is notational

Let

$$
\phi^{p, N}: E_{2}^{p, N}(A) \approx H^{p}\left(\Phi ; H^{N}(K ; A)\right)
$$

be the usual isomorphisms. On the right side we have nonhomogeneous $\Phi$ cochains in mind and think of $H^{N}(K ; A)$ as $H^{N}\left(V^{*}\right)$. Thus if $u \in E_{2}^{0, N}(A), \phi^{0, N}(u)$ is the cohomology class of a cocycle $f \in \operatorname{Hom}_{K}\left(C_{N}(K), A\right)$ and $\phi^{0, N}(u)$ is left fixed by $\Phi$, i.e. for each $\sigma \in \Phi, \sigma \cdot f$ is cohomologous to $f$. So there is

$$
h_{\sigma} \in \operatorname{Hom}_{K}\left(C_{N-1}(K), A\right)
$$

satisfying

$$
\delta h_{\sigma}=\sigma \cdot f-f \text {. }
$$

Recalling the chain homotopy $s_{*}$ and the cochain maps $j^{*}$ and $\psi^{*}$ defined in $\$ 1$, we can now state our result.

Proposition 3.1. A 2-cocycle (for $\Phi$ ) representing

$$
\phi^{2, N-1}\left(d_{2}(u)\right) \in H^{2}\left(\Phi ; H^{N-1}(K ; A)\right)
$$

is the function $X$ defined by

for $\sigma, \tau \in \Phi$.

$$
X(\sigma, \tau)=\sigma \cdot h_{\tau}-h_{\sigma \tau}-j^{N-1}\left\{\sigma \cdot\left[\left(\tau \cdot \psi^{N} f\right) \circ s_{N-1}\right]\right\} .
$$

Proof. We will use the interpretation of $d_{2}$ given in the remark in $\S 1$. Our first task then is to find an element of $L^{0, N} \oplus L^{1, N-1}$ representing $u \in E_{2}^{0, N}(A)$. First define $F \in L^{0, N}\left(=C^{0}\left(\Phi ; U^{N}\right)\right)$ by $F=\psi^{N} f$. Since

$$
d_{u} F=(-1)^{N} \psi^{N} \delta f=0,
$$

$F$ will serve as a "first component" for the representative of $u$. To find a "second component," we compute $d_{\Phi} F \in C^{1}\left(\Phi ; U^{N}\right)$. By definition

$$
\left(d_{\Phi} F\right)(\sigma)=\sigma \cdot F-F \in U^{N}=\operatorname{Hom}_{K}\left(C_{N}(G), A\right) .
$$


Using the machinery of the end of $\S 1$, we get

$$
\begin{aligned}
\sigma \cdot F & =\sigma \cdot\left(\psi^{N} f\right)=(\text { identity })^{N}\left[\sigma \cdot\left(\psi^{N} f\right)\right] \\
& =\left(j_{N} \psi_{N}-s_{N-1} \partial_{N}-\partial_{N+1} s_{N}\right)^{*}\left[\sigma \cdot\left(\psi^{N} f\right)\right] \\
& =\psi^{N} j^{N}\left[\sigma \cdot\left(\psi^{N} f\right)\right]-\delta\left(\left[\sigma \cdot\left(\psi^{N} f\right)\right] \circ s_{N-1}\right)-0,
\end{aligned}
$$

where $\partial_{*}$ is the boundary operator of $C_{N}(G)$.

The zero arises since $\delta f=0$. Now Proposition 1.2 says that the first term can be rewritten as $\psi^{N}(\sigma \cdot f)$. Hence

$$
\begin{aligned}
\sigma \cdot F & =\psi^{N}(\sigma \cdot f)-\delta\left(\left[\sigma \cdot\left(\psi^{N} f\right)\right] \circ s_{N-1}\right) \\
& =\psi^{N}\left(f+\delta h_{\sigma}\right)-\delta\left(\left[\sigma \cdot\left(\psi^{N} f\right)\right] \circ S_{N-1}\right) \text { by }(6) \\
& =F+\delta\left(\psi^{N} h_{\sigma}-\left[\sigma \cdot\left(\psi^{N} f\right)\right] \circ s_{N-1}\right), \text { i.e. } \\
d_{\Phi} F & =d_{u}\left(\psi^{N} h_{\sigma}-\left[\sigma \cdot\left(\psi^{N} f\right)\right] \circ s_{N-1}\right) .
\end{aligned}
$$

Define $Y \in L^{1, N-1}$ by

$$
\begin{aligned}
& Y(\sigma)=\psi^{N} h_{\sigma}-\left[\sigma \cdot\left(\psi^{N} f\right)\right] \circ s_{N-1}, \text { so } \\
& d_{\Phi} F=(-1)^{0} d_{u} Y .
\end{aligned}
$$

Thus by (1), $F+Y \in L^{0, N} \oplus L^{1, N-1}$ represents $u \in E_{2}^{0, N}(A)$, i.e. $Y$ is a second component.

By the remark of $\$ 1$, we may obtain a representative for $d_{2}(u)$ from $d_{\Phi} Y \in L^{2, N-1}=C^{2}\left(\Phi ; U^{N-1}\right)$.

We remark here that we do not want to know $d_{\Phi} Y$ itself, but rather $j^{N-1}\left(d_{\Phi} Y\right) \in C^{2}\left(\Phi ; V^{N-1}\right)$, and furthermore for a given $\sigma, \tau \in \Phi$, it will suffice to know the cohomology class in $H^{N-1}\left(V^{*}\right)$ of $j^{N-1}\left(d_{\Phi} Y(\sigma, \tau)\right)$. We define $X \in C^{2}\left(\Phi ; V^{N-1}\right)$ by

$$
X(\sigma, \tau)=j^{N-1}(\sigma \cdot Y(\tau)-Y(\sigma \tau)+Y(\sigma)) \text { for } \sigma, \tau \in \Phi,
$$

and we will show that the cohomology class of $X$ has the desired form.

Now by (7), $Y(\sigma)$ is the sum of what we will call a "first term" and a "second term"; so, therefore, is $X(\sigma, \tau)$. Using Proposition 1.2 again, we see that the first term of $X(\sigma, \tau)$ is $\sigma \cdot h_{\sigma}-h_{\sigma \tau}+h_{\sigma}$. The second term is

$$
\begin{aligned}
\sigma\left\{j^{N-1}\left(\left[\tau \cdot\left(\psi^{N} f\right)\right] \circ s_{N-1}\right)\right\} & -j^{N-1}\left(\left[\sigma \tau \cdot\left(\psi^{N} f\right)\right] \circ s_{N-1}\right) \\
& +j^{N-1}\left(\left[\sigma \cdot\left(\psi^{N} f\right)\right] \circ s_{N-1}\right),
\end{aligned}
$$

and since by Proposition $1.1, s_{N-1} j_{N-1}=0$, the second two terms of this expression vanish, and Proposition 3.1 now follows. 
REMARK. It is obvious from its definition that $\sigma \cdot h_{\tau}-h_{\sigma \tau}+h_{\sigma}$ is independent of the particular extension (i.e. of $\alpha$ ) and depends only on the $\Phi$-module structure of $K$. The second term does however depend on $\alpha$ since it involves the complex $C_{*}(G)$ more or less directly.

4. The second term. In this section we examine the second term defined in the previous section. To be more precise, we define $X_{1}$ and $X_{2}$ by

$$
X_{1}(\sigma, \tau)=\sigma \cdot h_{\tau}-h_{\sigma \tau}+h_{\sigma} \text { and }
$$

$$
X_{2}(\sigma, \tau)=j^{N-1}\left\{\sigma \cdot\left(\left[\tau \cdot\left(\psi^{N} f\right)\right] \circ s_{N-1}\right)\right\},
$$

so $X=X_{1}-X_{2}$. Note that for each $\sigma, \tau \in \Phi, X(\sigma, \tau)$ and $X_{1}(\sigma, \tau)$ are cocycles in $V^{N-1}=\operatorname{Hom}_{K}\left(C_{N-1}(K) ; A\right)$, and hence $X_{2}(\sigma, \tau)$ is also a cocycle.

In order to analyze the complicated compositions in (8), we introduce some more notation. Recall that $C_{k}(G)$ is a left $G$-module, and let $L_{\sigma-1}: C_{k}(G) \rightarrow C_{k}(G)$ be the homomorphism given by acting on the left with $\left(1, \sigma^{-1}\right) \in G(\sigma \in \Phi)$. This map commutes with the boundary operator in $C_{*}(G)$, but, of course, is not a $K$-module homomorphism. Let $A_{\sigma}: A \rightarrow A$ be the obvious map. Then (8) unravels to yield

$$
X_{2}(\sigma, \tau)=A_{\sigma} \circ A_{\tau} \circ f \circ \psi_{N} \circ L_{\tau-1} \circ s_{N-1} \circ L_{\sigma^{-1}} \circ j_{N-1}
$$

We include here a diagram which will perhaps aid the reader in following the discussion.

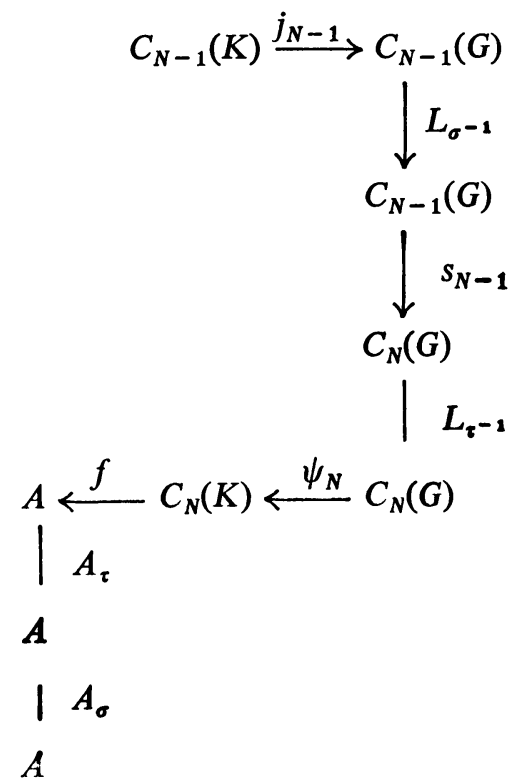


Define $\mathscr{H}_{l}(\sigma, \tau): C_{l}(K) \rightarrow C_{l+1}(K)$ by

$$
\mathscr{H}_{l}(\sigma, \tau)=B_{\sigma \tau} \circ \psi_{l+1} \circ L_{\tau-1} \circ s_{l} \circ L_{\sigma-1} \circ j_{l}
$$

where $B_{\rho}: C_{l}(K) \rightarrow C_{l}(K)$ for $\rho \in \Phi$ is the map that takes $\left(k_{0}, \cdots, k_{l}\right)$ into $\left(\rho \cdot k_{o}, \cdots, \rho \cdot k_{l}\right)$.

Proposition 4.1. For each $\sigma, \tau \in \Phi, \mathscr{H}_{l}(\sigma, \tau)$ is a K-module homomorphism.

Proof. The proposition follows easily from the fact that $s_{l}$ is a $K$-module homomorphism and that $L_{\sigma}$ and $B_{\rho}$ satisfy the relations

and

$$
L_{\sigma}(k \cdot x)=(\sigma \cdot k) L_{\sigma}(x) \text { for } k \in K \text { and } x \in C_{l}(G),
$$

$$
B_{\rho}(k \cdot y)=(\rho \cdot k) B_{\rho}(y) \text { for } k \in K \text { and } y \in C_{l}(K) .
$$

Proposition 4.2. Let $\Xi_{l}(\sigma, \tau): C_{l}(K) \rightarrow C_{l}(K)$ be the Z-homomorphism defined by

$$
\left(\Xi_{l}(\sigma, \tau)\right)\left(k_{0}, \cdots, k_{l}\right)=\left(k_{0}+\sigma \tau a\left(\tau^{-1}, \sigma^{-1}\right), \cdots, k_{l}+\sigma \tau a\left(\tau^{-1}, \sigma^{-1}\right)\right)
$$

where as in $\$ 1, a$ is normalized nonhomogeneous 2-cocycle representing $\alpha \in H^{2}(\Phi ; K)$. Then

(i) $\Xi_{l}(\sigma, \tau)$ is a K-module homomorphism

(ii) $\mathscr{H}_{*}(\sigma, \tau)$ is a chain homotopy between $\Xi_{*}(\sigma, \tau)$ and the identity $I$, i.e.

$$
\partial_{l+1} \mathscr{H}_{l}(\sigma, \tau)+\mathscr{H}_{l-1}(\sigma, \tau) \partial_{l}=I_{l}-\Xi_{l}(\sigma, \tau)
$$

Proof. (i) is entirely straightforward and will be omitted. For (ii) we get

$$
\begin{aligned}
\partial_{l+1} \mathscr{H}_{l}(\sigma, \tau) & +\mathscr{H}_{l-1}(\sigma, \tau)=B_{\sigma \tau} \circ \psi_{l+1} \circ L_{\tau-1} \circ\left(\partial_{l+1} s_{l}+s_{l-1} \partial_{l}\right) \circ L_{\sigma-1} j_{l} \\
& =B_{\sigma \tau} \circ \psi_{l} \circ L_{\tau-1}\left(j_{l} \circ \psi_{l}-I_{l}\right) \circ L_{\sigma^{-1}} \circ j_{l} \\
& =B_{\sigma \tau} \circ \psi_{l} \circ L_{\tau-1} \circ j_{l} \circ \psi_{l} \circ L_{\sigma-1} \circ j_{l}-B_{\sigma \tau} \circ \psi_{l} \circ L_{\tau-1} \circ L_{\sigma-1} \circ j_{l} .
\end{aligned}
$$

We indicate the value of the first of these homomorphisms on $\left(k_{0}, \cdots, k_{l}\right)$ diagramatically,

$$
\begin{gathered}
j_{l}:\left(k_{0}, \cdots, k_{l}\right) \stackrel{j_{l}}{\rightarrow}\left(\left(k_{0}, 1\right), \cdots,\left(k_{l}, 1\right)\right) \stackrel{\left(L \sigma^{-1}\right)}{\longrightarrow}\left(\left(\sigma^{-1} \cdot k_{0}, \sigma^{-1}\right), \cdots,\left(\sigma^{-1} \cdot k_{l}, \sigma^{-1}\right)\right) \\
\left(\tau^{-1} \sigma^{-1} k_{0}, \tau^{-1}\right), \cdots\left(\tau^{-1} \sigma^{-1} k_{l}, \tau^{-1}\right) \stackrel{L(\tau-1)}{\stackrel{L}{\leftarrow}}\left(\left(\sigma^{-1} k_{0}, 1\right), \cdots,\left(\sigma^{-1} k_{l}, 1\right)\right) \stackrel{j_{l}}{\leftarrow}\left(\sigma^{-1} k_{0}, \cdots, \sigma^{-1} k_{l}\right) \\
\qquad \psi_{l} \\
\left(\tau^{-1} \sigma^{-1} k_{0}, \cdots, \tau^{-1} \sigma^{-1}, k_{l}\right) \stackrel{B_{\sigma \tau}}{\longrightarrow}\left(k_{0}, \cdots, k_{l}\right) .
\end{gathered}
$$

Proceeding in a similar fashion for the second homomorphism, we get 


$$
\begin{gathered}
\left(k_{0}, \cdots, k_{l}\right) \stackrel{j_{l}}{\rightarrow}\left(\left(k_{0}, 1\right), \cdots,\left(k_{l}, 1\right)\right) \stackrel{L\left(\sigma^{-1}\right)}{\rightarrow}\left(\left(\sigma^{-1} k_{0}^{-1} \sigma^{-1}\right), \cdots,\left(\sigma^{-1} k_{l}, \sigma^{-1}\right)\right) \\
\left(\left(\tau^{-1} \sigma^{-1} \cdot k_{0}+a\left(\tau^{-1}, \sigma^{-1}\right), \tau^{-1} \sigma^{-1}\right)\right), \cdots,\left(\tau^{-1} \sigma^{-1} \cdot k_{l}+a\left(\tau^{-1}, \sigma^{-1}\right), \tau^{-1} \sigma^{-1}\right) \\
\downarrow^{\downarrow} \psi_{l} \\
\left(\tau^{-1} \sigma^{-1} k_{0}+a\left(\tau^{-1}, \sigma^{-1}\right), \cdots, \tau^{-1} \sigma^{-1} k_{l}+a\left(\tau^{-1}, \sigma^{-1}\right)\right) \\
\downarrow \\
\left(k_{0}+\sigma \tau \cdot a\left(\tau^{-1}, \sigma^{-1}\right), \cdots, k_{l}+\sigma \tau \cdot a\left(\tau^{-1}, \sigma^{-1}\right)\right),
\end{gathered}
$$

which concludes the proof.

Now using the notation of $\S 3$, we can state an extension of Proposition 3.1.

Proposition 4.3. Let $\mathscr{H}_{l}^{\prime}(\sigma, \tau): C_{l}(K) \rightarrow C_{l+1}(K)$ be any chain homotopy between $I_{l}$ and $\Xi_{l}(\sigma, \tau)$. Then a 2-cocycle representing $\phi^{2, N-1}\left(d_{2}(u)\right)$ is the function $W$ defined by

$$
W(\sigma, \tau)=\sigma \cdot h_{\tau}-h_{\sigma \tau}+h_{\sigma}-[(\sigma \tau) \cdot f] \circ \mathscr{H}_{N-1}^{\prime}(\sigma, \tau) .
$$

Proof. Note that $[\sigma \cdot \tau \cdot f] \circ \mathscr{H}_{N-1}(\sigma, \tau)=X_{2}(\sigma, \tau)$, so that in view of the previous discussion, the only point requiring justification is the substitution of an arbitrary $\mathscr{H}_{*}^{\prime}(\sigma, \tau)$ for the specific $\mathscr{H}_{*}(\sigma, \tau)$. But general theory tells us that for any $\mathscr{H}_{*}^{\prime}$ there exist $K$-module homomorphisms

$$
T_{l}(\sigma, \tau): C_{l}(K) \rightarrow C_{l+2}(K)
$$

satisfying

$$
\partial_{l+2} T_{l}(\sigma, \tau)+T_{l-1}(\sigma, \tau) \partial_{l}=\mathscr{H}_{l}(\sigma, \tau)-\mathscr{H}_{l}(\sigma, \tau) .
$$

Thus if $g$ is an $N$-cocycle $g \circ \mathscr{H}_{N-1}(\sigma, \tau)=g \circ \mathscr{H}_{N-1}^{\prime}(\sigma, \tau)+0+a$ coboundary. Since $(\sigma \tau \cdot f)$ is a cocycle, the result follows.

5. Pontryagin products. Before continuing with the main argument, we pause to consider Pontryagin multiplication in $H_{*}(K ;)$. Our basic reference for this is [2]. We then will relate the chain homotopies $\mathscr{H}_{l}^{\prime}(\sigma, \tau)$ of the previous section with the Pontryagin product.

The basic element in the definition of Pontryagin multiplication is the chain map

$$
P: C_{*}(K) \otimes_{\mathrm{z}} C_{*}(K) \rightarrow C_{*}(K)
$$

which satisfies

(i) $\boldsymbol{P}\left(k_{1} x_{1} \otimes k_{2} x_{2}\right)=\left(k_{1}+k_{2}\right) \boldsymbol{P}\left(x_{1} \otimes x_{2}\right)$ for any $k_{1}, k_{2} \in K$ and $x_{1}, x_{2} \in C_{*}(K)$, and 
(ii) the diagram

is commutative.

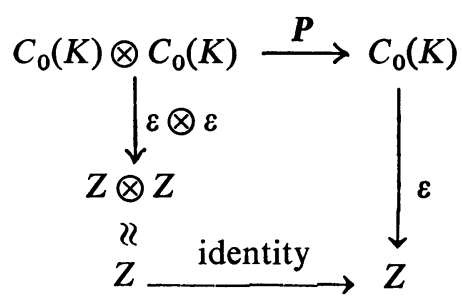

Such maps $\boldsymbol{P}$ aways exist if $K$ is abelian (see [2] for more details). We wish to point out the following slight sharpening of the existence theorem for such a $\boldsymbol{P}$. Its proof is no more difficult than the original and will be omitted.

Proposition 5.1. A map P can be chosen so that in addition to satisfying (i) and (ii), it also satisfies

(iii) if $k \in K$ (and hence $(k) \in C_{0}(K)$ ) and $x_{n} \in C_{n}(K)$, then

$$
\boldsymbol{P}\left((k) \otimes x_{n}\right)=k \cdot x_{n} \text {. }
$$

In what follows we always assume $\boldsymbol{P}$ satisfies (i), (ii), and (iii).

For $k \in K,(k) \in C_{0}(K)$ and $(0, k) \in C_{1}(K)$. We define maps

$$
G_{n}(k): C_{n}(K) \rightarrow C_{n}(K): x_{n} \rightarrow \boldsymbol{P}\left((k) \otimes x_{n}\right)
$$

and

$$
D_{n}(k): C_{n}(K) \rightarrow C_{n+1}(K): x_{n} \rightarrow \boldsymbol{P}\left((0, k) \otimes x_{n}\right) .
$$

Proposition 5.2. $G_{n}(k)$ and $D_{n}(k)$ are K-module homomorphisms and

$$
\partial_{n+1} D_{n}(k)+D_{n-1}(k) \partial_{n}=G_{n}(k)-I_{n} .
$$

Proof. The first statement is trivial, and the second follows from

$$
\begin{aligned}
& \partial_{n+1} \boldsymbol{P}\left((0, k) \otimes x_{n}\right)+\boldsymbol{P}\left((0, k) \otimes \partial x_{n}\right)=\left[\boldsymbol{P}\left(\partial(0, k) \otimes x_{n}\right)+(-1)^{1} \boldsymbol{P}\left((0, k) \otimes \partial x_{n}\right)\right] \\
& +\boldsymbol{P}\left((0, k) \otimes \partial x_{n}\right)=\boldsymbol{P}\left((0) \otimes x_{n}\right)-\boldsymbol{P}\left((k) \otimes x_{n}\right)=x_{n}-k \cdot x_{n}=I_{n}\left(x_{n}\right)-\left[G_{n}(k)\right]\left(x_{n}\right)
\end{aligned}
$$

since $\boldsymbol{P}$ is a chain map.

COROLlaRY 5.3. If we use the notation of the previous section and put $b(\sigma, \tau)=\sigma \tau \cdot a\left(\tau^{-1}, \sigma^{-1}\right)$, then we may choose $\mathscr{H}_{n}^{\prime}(\sigma, \tau)=-D_{n}(b(\sigma, \tau))$.

Now we restrict back to the situation in $\S 2$ and take $A$ to be $F$-free. We will identify $H_{1}(K ; Z)$ with $K$ by the canonical isomorphism. We remark that a cycle corresponding to $k \in K$ is $1 \otimes_{K}(0, k) \in Z \otimes_{K} C_{1}(K)$. Let

$$
p_{n}: H^{n}(K ; A) \rightarrow \operatorname{Hom}_{F}\left(H_{n}(K ; F), A\right)
$$

be the homomorphism appearing in the universal coefficient theorem. Pontryagin multiplication gives us a map 


$$
H_{1}(K ; Z) \otimes_{Z} H_{n-1}(K ; \boldsymbol{F}) \rightarrow H_{n}\left(K, Z \otimes_{Z} \boldsymbol{F}\right) \approx H_{n}(K ; \boldsymbol{F}),
$$

or equivalently a homomorphism

$$
\boldsymbol{P}_{*}^{\prime}: K \rightarrow \operatorname{Hom}_{F}\left(H_{n-1}(K ; \boldsymbol{F}), H_{n}(K ; \boldsymbol{F})\right) .
$$

With this preparation we can now state the following proposition:

Proposition 5.4. Let $u \in H^{n}(K ; A)$ be represented by the cocyle $g \in \operatorname{Hom}_{K}\left(C_{n}(K), A\right)$. Then $g \circ D_{n-1}(k) ; C_{n-1}(K) \rightarrow A$ is a cocycle and thereby defines an element $v \in H^{n-1}(K ; A)$. We have

$$
p_{n-1}(v)=p_{n}(u) \circ \boldsymbol{P}_{*}^{\prime}(k) \text {. }
$$

Proof. $\delta\left(g \circ D_{n-1}(k)\right)=g \circ D_{n-1}(k) \circ \partial_{n}=g \circ\left[G_{n}(k)-I_{n}-\partial_{n+1} \circ D_{n}(k)\right]=g \circ G_{n}(k)-$ $g-0$ since $g$ is a cocycle. Since $A$ is $K$-trivial, $g \circ G_{n}(k)=g$, so $g \circ D_{n-1}(k)$ is a cocycle. For the second half of the assertion we introduce the following notation: If $x_{i} \in A \otimes_{K} C_{i}(K)$ or $\operatorname{Hom}_{K}\left(C_{i}(K), A\right)$ is a cycle or cocycle, $\hat{x}_{i}$ is its homology or cohomology class. Take $y \otimes_{K} c_{n-1} \in A \otimes_{K} C_{n-1}(K)$ to be a cycle, then

$$
\begin{aligned}
{\left[p_{n-1}(v)\right]\left(\left\langle y \otimes_{K} c_{i-1}\right\rangle\right) } & =\left[p_{n-1}\left(\left\langle g \circ G_{n-1}(k)\right\rangle\right)\right]\left(y \otimes_{K} c_{i-1}\right) \\
& =y \cdot\left[\left(g \circ G_{n-1}(k)\right)\left(c_{n-1}\right)\right]=y \cdot\left[g\left(\boldsymbol{P}^{\prime}\left((0, k) \otimes c_{n-1}\right)\right)\right] \\
& =y \cdot\left[\left(g \circ \boldsymbol{P}_{*}^{\prime}(k)\right)\left(c_{n-1}\right)\right)=p_{n}(u) \circ \boldsymbol{P}_{*}^{\prime}(k)\left(\left\langle y \otimes_{K} c_{i-1}\right\rangle\right) .
\end{aligned}
$$

6. The Main Theorem. For the convenience of the reader, we briefly recall the situation and notation of $\S 2$. Each $\alpha \in H^{2}(\Phi ; K)$ gives rise to an extension $G$ and hence to a spectral sequence $\hat{E}_{r}^{p, q}\left(=E_{r}^{p, q}\left(H_{N}(K ; F)\right)\right)$ and to a differential $d_{2}: \hat{E}_{2}^{0, N} \rightarrow \hat{E}_{2}^{2, N-1}$ which, by a canonical isomorphism, induces

$$
D_{\alpha}: H^{0}\left(\Phi ; H^{N}\left(K ; H_{N}(K ; \boldsymbol{F})\right)\right)=H^{2}\left(\Phi ; H^{N-1}\left(K ; H_{N}(K ; \boldsymbol{F})\right)\right) .
$$

We also have a particular element $f^{N} \in H^{0}\left(\Phi ; H^{N}\left(K ; H_{N}(K ; F)\right)\right)$. Since we have assumed that $H_{i}(K ; F)$ is $F$-free for all $i, p_{i}: H^{i}\left(K ; H_{N}(K ; F)\right) \rightarrow \operatorname{Hom}_{F}\left(H_{i}(K ; F)\right.$, $\left.H_{N}(K ; F)\right)$ is an isomorphism for all $i$.

As remarked in the last section, Pontryagin multiplication gives a homomorphism of $\Phi$-modules by the following composition:

$K \approx H_{1}(K ; Z) \stackrel{P_{*}^{\prime}}{\longrightarrow} \operatorname{Hom}_{F}\left(H_{N-1}(K ; F), H_{N}(K ; F)\right) \stackrel{p_{N-1}^{-1}}{\approx} H^{N-1}\left(K ; H_{N}(K ; F)\right)$.

In this section we will denote this composition by $\boldsymbol{P}$ and let $\boldsymbol{P}^{*}$ denote the induced map

$$
\boldsymbol{P}_{*}: H^{i}(\Phi ; K) \rightarrow H^{i}\left(\Phi ; H^{N-1}\left(K ; H_{N}(K ; \boldsymbol{F})\right)\right) \text {. }
$$

TheOREM 6.1. $D_{\alpha}\left(f^{N}\right)=D_{0}\left(f^{N}\right)-\boldsymbol{P}_{*}(\alpha)$. 
Proof. $\$ 5$ gives a computation of a cocycle $Y$ which represents $D_{\alpha}\left(f^{N}\right)$. We have

$$
Y(\sigma, \tau)=\sigma \cdot h_{\tau}-h_{\sigma \tau}+h_{\sigma}+\left[(\sigma \tau) \cdot f^{N}\right] \circ D_{n-1}(b(\sigma, \tau)) .
$$

Proposition 5.4 with $A=H_{n}(K ; F)$ together with the definition of $f^{N}$ implies that the term $\left[(\sigma \tau) \cdot f^{N}\right] \circ D_{n-1}(b(\sigma, \tau))$ represents $\boldsymbol{P}_{*}(\beta)$ where $\beta \in H^{2}(\Phi ; K)$ is the class of $b$. The other term in $Y$ is independent of $\alpha$ and is easily seen to represent $D_{0}\left(f^{N}\right)$. Hence the theorem will follow from the following lemma.

LEMMA 6.2. $\beta=-\alpha$.

Proof. Define $c: \Phi \rightarrow K$ by $c(\sigma)=a\left(\sigma, \sigma^{-1}\right)$. It is a straightforward computation to see that $b+a=\delta c$, which suffices to prove the lemma.

REMARK. Note that Theorem 6.1 together with Proposition 2.2 yields the main theorem of the introduction.

7. The split extension. We consider the split extension $G$ which corresponds to $\alpha=0 \in H^{2}(\Phi ; K)$. In this case $G$ is the semidirect product of $K$ and $\Phi$. The general considerations of the previous discussion lead us to consider the elements

$$
v^{N}=D_{0}\left(f^{N}\right) \in H^{2}\left(\Phi ; H^{N-1}\left(K ; H_{N}(K ; \boldsymbol{F})\right)\right) .
$$

These classes depend only on the structure of $K$ as a $\Phi$-module, and so we purpose to call them the characteristic classes of the $\Phi$-module $K$. It is easy to see that they vanish for a trivial $\Phi$-module, and, roughly speaking, they provide some mcasure of the difference between the cohomology of the semidirect product and the direct product. In general we know very little about these classes. They are not always zero - an example will be given later. We have, however, the following fact:

Proposition 7.1. For any $\Phi$-module $K, v^{1}=0$.

Proof. Since we have a homomorphism $s: \Phi \rightarrow G$ with $\rho s$ equal the identity, naturality shows that $\rho^{*}: H^{*}(\Phi ; A) \rightarrow H^{*}(G ; A)$ is a monomorphism, and, in fact, $s^{*} \rho^{*}=(\rho s)^{*}$ is the identity. Now the image of $\rho^{*}$ corresponds to the subgroup $E_{\infty}^{*, 0}(A)$ which is a quotient group of $H^{*}\left(\Phi ; H^{\circ}(K ; A)\right) \approx H^{*}(\Phi ; A)$. Thus $E_{\infty}^{*, 0}(A)=E_{2}^{*, 0}(A)$. In particular, $d_{2}$ must have image 0 for all elements of $E_{2}^{*, 1}$. But $D_{0}$ is defined by $d_{2}$ in an appropriate spectral sequence.

REMARK. Putting together this last proposition with 2.2 and 6.1 , we obtain a formula for $d_{2}: E_{2}^{*, 1}(\boldsymbol{F}) \rightarrow E_{2}^{*, 0}(\boldsymbol{F})$. This should be compared with [7, p. 133].

We now define the classes $v^{N}$ without the use of a spectral sequence.

Let $C_{*}(K)$ be as defined in $\S 1$, and now denote $\operatorname{Hom}_{K}\left(C_{t}(K), A\right)$ by $C^{i}(K ; A)$. As usual, this is a $\Phi$-module and $\delta: C^{i}(K ; A) \rightarrow C^{i+1}(K ; A)$ is a $\Phi$-module homomorphism. Thus we obtain the following usual exact sequences of $\phi$-modules:

$$
0 \rightarrow B^{i}(K ; A) \rightarrow Z^{i}(K ; A) \stackrel{\pi_{i}}{\rightarrow} H^{i}(K ; A) \rightarrow 0,
$$




$$
0 \rightarrow Z^{i}(K ; A) \rightarrow C^{i}(K ; A) \stackrel{\delta^{i}}{\rightarrow} B^{i+1}(K ; A) \rightarrow 0 .
$$

We will denote the Bockstein (or connecting) homomorphism associated to (10), by $\Delta_{i}^{0}$ and to $(11)_{i}$ by $\Delta_{i}^{1}$.

Let $D_{0}: H^{p}\left(\Phi ; H^{q}(K ; A)\right) \rightarrow H^{p+2}\left(\Phi ; H^{q-1}(K ; A)\right)$ be the homomorphism corresponding to $d_{2}: E_{2}^{p, q}(A) \rightarrow E_{2}^{p+2, q-1}(A)$ in the spectral sequence arising from the split extension, and assume $A$ is an $\boldsymbol{F}$-module.

Proposition 7.2. Let $u \in H^{0}\left(\Phi, H^{N}(K ; A)\right)$. Then

$$
D_{0}(u)=\pi_{N-1} \circ \triangle_{N-1}^{1} \circ \triangle_{N}^{0}(u) \in H^{2}\left(\Phi ; H^{N-1}(K ; A)\right) .
$$

Proof. Since $H_{N-2}(K ; F)$ is $F$-projective, $\operatorname{Ext}_{\boldsymbol{F}}\left(H_{N-2}(K ; F), A\right)=0$, so $H^{N-1}(K ; A) \approx \operatorname{Hom}_{F}\left(H_{N-1}(K ; F), A\right)$. Furthermore the computation of $\S 3$ shows that $D_{0}(u)$ can be represented by $\sigma \cdot h_{\tau}-h_{\sigma \tau}+h_{\tau}$ plus a term that vanishes since $\alpha=-\beta=0$ where $\delta h_{\sigma}=\sigma \cdot f-f$ and $f$ represents $u$. The proposition now follows upon chasing through the definition of the Bockstein homomorphisms involved.

REMARK. If we take $A=H_{N}(K ; \boldsymbol{F})$, we obtain the promised procedure for computing $v^{n}=D_{0}\left(f^{N}\right)$. Furthermore this provides an alternative proof that $v^{1}=0$, since $H_{1}(K ;)$ is trivial $K$-module, and so $B^{1}\left(K ; H_{1}(K ; F)\right)=0$.

8. Some examples. It seems appropriate at this point to give some examples. As must be obvious to the reader, a computation of the characteristic classes $v^{N}$ is extremely difficult even if the group $K$ is not complicated, and its cohomology is known. So far, all descriptions of the $v^{N}$ depend on the specific complex $C_{*}(K)$. Essentially, this is because $\Phi$ acts naturally on this complex. This complex is, however, not always convenient for calculations. In fact, explicit computations of the cohomology of $K$ usually hinge on replacing $C_{*}(K)$ by a more "efficient" complex. What is needed is a set of theorems that describes the classes $v^{N}$ in terms of any resulotion. There is no difficulty in finding such theorems. The resulting formulae will be given explicitly in [9]. At the moment we state without proof the results of some such computations. The proofs will also appear in [9].

EXAMPLE 1. $K=Z_{8}=$ the cyclic group of order eight; $\Phi=Z_{2}=$ the group with two elements. $\Phi$ acts on $K$ by multiplication by five; i.e. if $\sigma$ is the nontrivial element of $\Phi$ then $\sigma \cdot x=5 \cdot x$. We choose $A=\boldsymbol{F}=$ the field of two elements. Thus $H_{i}(K, F)=Z_{2}$ in all dimensions. In this case $v^{2}=D_{0}\left(f^{2}\right) \neq 0$. This is the simplest example we know in which a characteristic class is nonzero - even when the final coefficients are a field.

EXAMPLE 2. In this example we again have $v^{2} \neq 0$. This time $\Phi$ is $Z_{2} \oplus Z_{2}$ and $K$ is the dual of the augmentation ideal in $Z[\Phi]$, i.e. $K$ is thus a free abelian group of rank 3 . We choose for final coefficients the group $Z$. Thus

$$
v^{2} \in H^{2}\left(\Phi ; H^{1}\left(K ; H_{2}(K ; Z)\right)\right)
$$


We show in [9] that this group is $Z_{4}$ and $v^{2}$ is twice a generator. Note that $v^{2}$ has order 2 . We show in [9] that $v^{i}$ always has order 2 (any $i$ ) if $K$ is $Z$-free.

EXAMPLE 3. We take $\Phi=Z_{p}=$ a cyclic group of prime order, and restrict attention to the case where $K$ is free abelian of finite rank as an abelian group. All module structures for this situation are described in [6]. Making use of this explicit detailed knowledge of these modules we can prove that $v^{i}=0$ for all $i ; v^{i} \in H^{2}\left(\Phi ; H^{i-1}\left(K, H_{i}(K, Z)\right)\right)$.

9. An application. References for this section are [1], [4] and especially [5]. If $X$ is a compact flat riemannian manifold and $\pi=\pi_{1}(x)$, then $X$ is a $K(\pi, 1)$, so $H^{*}(X) \approx H^{*}(\pi)$. Furthermore $\pi$ is torsion free and satisfies an exact sequence

$$
0 \rightarrow K \rightarrow \pi \rightarrow \Phi \rightarrow 1
$$

where $K$ is free abelian (of rank equal to the dimension of $X$ ) and normal and maximal abelian in $\pi ; \Phi$ is finite and isomorphic to the holonomy group of $X$. In this section we use our Main Theorem to derive some facts about the cohomology of such manifolds when $\Phi$ is cyclic and, in particular, of prime order.

Now we suppose $\Phi$ is $Z_{m}$, a cyclic group of prime order $m$, and that $K=L \oplus Z$ as a $\Phi$-module, i.e. $K$ contains a direct summand $Z$ on which $\Phi$ acts trivially. Furthermore, if $\alpha \in H^{2}(\Phi ; K)$ classifies $\left(\begin{array}{c}* \\ *\end{array}\right)$, we may assume $\alpha$ is in the image of the map: $H^{2}(\Phi ; Z) \rightarrow H^{2}(\Phi ; K)$ given by the inclusion.

THEOREM 9.1. If $E_{r}^{i, j}$ is the spectral sequence for $\left(\begin{array}{c}* \\ *\end{array}\right)$ with coefficients $Z$, then

$$
E_{3}^{0, j} \approx H^{0}\left(Z_{m} ; H^{j}(K ; Z)\right) ; E_{3}^{1, j} \approx H^{1}\left(Z_{m} ; H^{j-1}(L ; Z)\right)
$$

and $E_{3}^{i, j}=0$ for $i>1$.

We sketch the proof as follows: If $M$ is a $Z_{m}$-module, we let $M^{*}$ be the $Z_{m}$-module $\operatorname{Hom}_{Z}(M, Z)$. Then it is well known that $H^{j}(M ; Z) \approx \wedge^{j} M^{*}$ as a $Z_{m}$-module where the exterior power is taken over $Z$ and $Z_{m}$ acts "diagonally." Therefore

$$
H^{j}(K ; Z) \approx H^{j}(L ; Z) \oplus Z \wedge H^{j-1}(L ; Z)
$$

where " $\wedge$ " can be thought of as Pontryagin multiplication in $H^{*}(M ; Z)$. Hence

$$
\begin{aligned}
E_{2}^{i, j} & \approx H^{i}\left(Z_{m} ; H^{j}(L ; Z)\right) \oplus H^{i}\left(Z_{m} ; Z \wedge H^{j-1}(L ; Z)\right) \text { and } \\
E_{2}^{i+2, j-1} & \approx H^{i}\left(Z_{m} ; H^{j-1}(L ; Z)\right) \oplus H^{i}\left(Z_{m} ; Z \wedge H^{j-2}(L ; Z)\right),
\end{aligned}
$$

where we have used the fact that the cohomology of $Z_{m}$ is periodic of order two. Now by the Main Theorem, $d_{2}: E_{2}^{i, j} \rightarrow E_{2}^{i+2, j-1}$ is given by cupping with $\pm \alpha$ and since, roughly speaking, $\alpha$ is in the summand $Z$ of $M, d_{2}$ maps $H^{i}\left(Z_{m} ; H^{j}(L ; Z)\right)$ onto 0 , and takes the summand $H^{i}\left(Z_{m} ; Z \wedge H^{j-1}(L ; Z)\right)$ 
isomorphically onto $H^{l}\left(Z_{m} ; H^{j-1}(L ; Z)\right)$. Therefore, away from the "left edge" everything dies, and the theorem now follows trivially from (17), after noting that $E_{2}^{0, j}$ is free so neither part dies there.

COROLlary 9.2. Under the above hypothesis, the cohomology of $\pi$ satisfies an exact sequence

$$
0 \rightarrow T^{*} \rightarrow H^{*}(\pi ; Z) \rightarrow F^{*} \rightarrow 0 .
$$

where $T^{*}$, the torsion ideal of $H^{*}(\pi, Z)$, is a finite direct sum of $Z_{m}$ 's and has trivial cup products while $F^{*}$ is a free abelian group which is a subalgebra of an exterior algebra.

The proof follows trivially from the observation that $E_{3}=E_{\infty}$ by position.

REMARK. It follows from [4] that if $\pi$ is torsion free, $K$ can always be written as $L \oplus Z$, and furthermore that $\alpha$ is in the image of the map: $H^{2}\left(Z_{p} ; Z\right) \rightarrow H^{2}\left(Z_{p} ; K\right)$. Hence Corollary 9.2 applies and tells us that the cohomology of a compact riemannian manifold whose holonomy group is cyclic of prime order satisfies (18). For an alternative approach see [5]. We should remark that this problem provided the original motivation for this paper.

\section{REFERENCES}

1. L. Auslander and M. Kuranishi, On the homology group of locally Euclidean spaces, Ann. of Math. 65 (1957), 411.

2. Seminaire H. Cartan, Cohomologie des groupes, suite spectrale, Faisceaux: 1950/1951, Exposé No. 4.

3. H. Cartan and S. Eilenberg, Homological algebra, Princeton Univ. Press, Princeton, N.J., 1956.

4. L. S. Charlap, Compact flap riemannian manifolds. I, Ann. of Math. 81 (1965), 15.

5. L. S. Charlap and A. T. Vasquez, Compact flat riemannian manifolds. II, Amer. J. Math. 87 (1965), 551.

6. C. W. Curtis and I. Reiner, Representation theory of finite groups and associative algebras, Interscience, New York, 1962.

7. R. Godement, Topologie algébrique et théorie des faisceaux, Actualités Sci. Ind. No. 1252, Hermann, Paris, 1958.

8. G. Hochschild and J.-P. Serre, The cohomology of group extensions, Trans. Amer. Math. Soc. 74 (1953), 110.

9. L. S. Charlap and A. T. Vasquez, Characteristic classes for modules over groups (to appear).

INSTITUTE FOR ADVANCED StUdY,

Princeton, NeW Jersey 